\title{
The evaluation of reliability of hydraulic conductivity and specific storage structures estimated by Self -potential inversion.
}

\author{
Yusuke OZAKI ${ }^{1,2,3}$, Hitoshi MIKADA ${ }^{1}$, Tada-nori GOTO ${ }^{1,4}$ and Junichi TAKEKAWA \\ ${ }^{1}$ Dept. of Civil and Earth Res. Eng., Kyoto University \\ ${ }^{2}$ JSPS research Fellow. \\ ${ }^{3}$ Now, Japan Atomic Energy Agency \\ ${ }^{4}$ Now, Dept. of Urban Management, Kyoto University
}

\begin{abstract}
The objective of this study is the evaluation of reliability of estimated hydraulic conductivity and specific storage structure from self-potential (SP) data under two difficult conditions. As one of difficult condition, the lack of flux data is assumed. This situation is assumed because the sampling rate of flux data would become lower than the SP data when the monitoring data is focused on. The effect of metal casing pipe is evaluated as the other difficult condition case. Our numerical tests show that only the estimation result of hydraulic conductivity structure can be reliable when the sampling rate of the flux data is too low. It is also demonstrated that the metal casing changes the value of estimation results of hydraulic conductivity and specific storage around the casing pipe. We also demonstrate that the effect of metal casing pipe can be removed by the inversion scheme proposed in this study.
\end{abstract}

\section{TITLE PAGE}

The Self-potential (SP) inversion scheme with the flux data has been proposed for the estimation of hydrogeophysical parameters. Soueid Ahmed et al. (2014) developed the estimation method of hydraulic conductivity structure by the steady state analysis of SP and head data. Simultaneous estimation of hydraulic conductivity and specific storage structure by the transient SP inversion has also been developed (Ozaki et al., 2014). These SP inversion schemes are efficient estimation method of hydrogeophysical parameters because the SP data is possible to acquire on the ground surface and the number of wells for the estimation can be reduced.

Although the SP inversion is useful tool for the estimation of hydrogeophysical parameters, the practical application of SP inversion remains to be difficult because SP signals are vulnerable to the variable factors, such as electrical conductivity structure. The deviation of the estimation image of hydraulic conductivity structure due to the uncertainty of the electrical conductivity structure is evaluated by Soueid Ahmed et al. (2014). The effect of high electrical conductive metal casing to the SP distribution cannot be ignored, too. The effect of casing pipe is indicated by several authors (e.g.,
Darnet et al., 2002). The other uncertainty is the difference of the data quality between SP and flux data. Although the transient SP inversion enables to analyze the time lapse SP data, the quality of SP data is not always same as that of flux data, especially in case of monitoring data. The sampling rate of flux data would be lower than that of SP data because of the difference of difficulty of the measurements.

In this study, we evaluate the effect of existence of metal casing pipe on the inversion results. The effect to input the insufficient flux data to the inversion is also evaluated. The purpose of this study is the evaluation of the reliability of inversion images under these two conditions

\section{Method and Theory}

In this study, we perform the numerical tests for the evaluation of the reliability of estimated hydraulic conductivity and specific storage structures. The inversion method used in this study is based on the optimization of the objective function defined by Tikhonov regularization with the smooth constraint. The Gauss-Newton algorithm is employed for the optimization. The details of the inversion scheme are in Ozaki et al. (2014). The effect of the unsaturated zone on the SP 
distribution is ignored in this study.

The subsurface models of hydraulic conductivity and specific storage structures used in this study are generated with the geostatistical software SGeMS (Deutsch and Journel, 1992). The 100 random values at the 100 random locations are selected for the generation. Figure 1 shows the models of hydraulic conductivity and specific storage structures for the numerical tests.

\section{Casing effect}

In this section, we investigate the effect of metal casing in the borehole for the injection or pumping on the SP inversion results. The metal casing is high electrical conductivity structure. This high conductive structure conducts the SP signal without the decay of potential from the deep subsurface to the ground surface. This effect disturbs the SP distribution observed on the ground surface (Darnet et al., 2002; Titov et al., 2005). The analysis results of the SP distribution affected by this structure would be biased if the effect is ignored.

In this study, the electrical conductivity of the casing pipe is assumed to be infinite; the area of the infinitely conductive zone is assumed to be equipotential. We employ the modified conjugate gradient $(\mathrm{CG})$ method for the calculation of the electrical potential including the equipotential zone. The values corresponding to the equipotential zone in all gradient vectors in the $\mathrm{CG}$ method are constrained to the same values for the calculation of the SP including the equipotential zone.

Figure 2 shows the SP distribution on the ground surface considering and ignoring the effect of existence of metal casing pipe. The SP distributions are in steady states during pumping. The location of

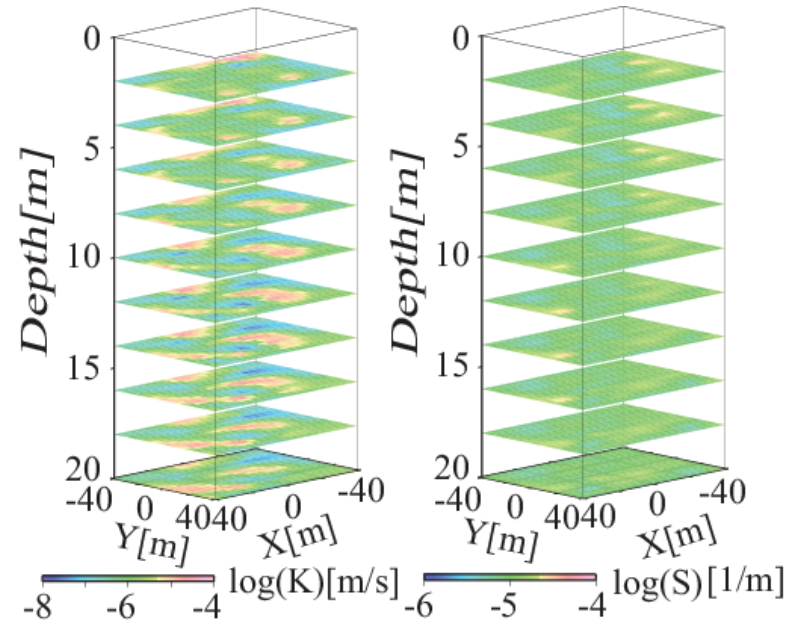

Figure 1 Model of hydraulic conductivity structure (left) and specific storage (right). The borehole is located at the centor of models $(\mathrm{X}=0 \mathrm{~m}, \mathrm{Y}=0 \mathrm{~m})$.

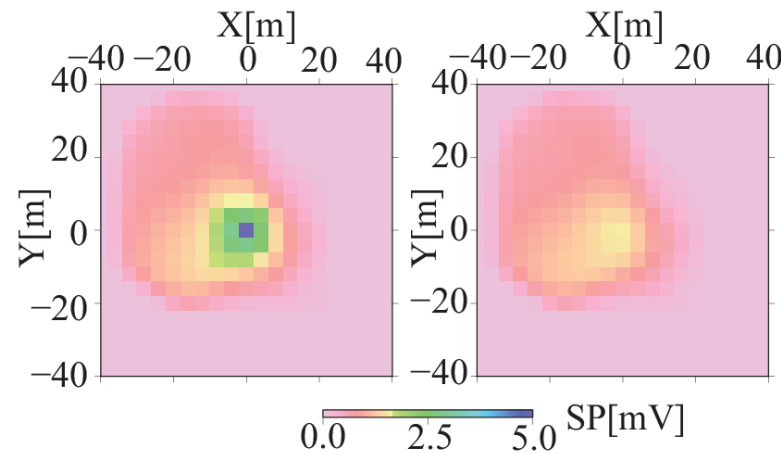

Figure 2 The SP distributions on the ground surface. The distributionos SP considering the effect of metal casing pipe (left) and ignoring the effect (right) are shown. The metal casing is located at the centor of the model from the ground surface to the depth of $10 \mathrm{~m}$ in the models in Figure1.

the pumping point is the center of the model in Figure $1(\mathrm{X}=0 \mathrm{~m}, \mathrm{Y}=0 \mathrm{~m})$ and the depth of pumping point is $10 \mathrm{~m}$. The pumping rate is $0.1 \mathrm{Ls}^{-1}$ and the uniform electrical conductivity structure of 0.01 $\mathrm{Sm}^{-1}$ is assumed. The trend of the SP distribution in both cases is similar except for the vicinity of borehole which is the center of the distribution. The SP around the borehole becomes high value when the effect of casing pipe is considered. This is because that the high electrical potential at the pumping point conducts to the ground surface through the metal casing. However, the area where the effect of existence of casing pipe appears is limited only around the borehole in this case.

To investigate the effect of the casing pipe on the inversion results, we analyze the SP distribution affected the effect of metal casing (Figure2 right) with the transient inversion considering and ignoring the effect. $36 \mathrm{SP}$ variations are input to the inversions. Figure 3 shows the inversion results. When the effect of the casing pipe is included in the inversion scheme, the correct subsurface images of hydraulic conductivity and specific storage structures are reproduced. When the casing effect is ignored, the hydraulic conductivity and specific storage around the casing pipe are estimated more highly and lowly than those of true model, respectively. The casing pipe in the pumping or injection well works so that it leads the high potential at the pumping point without decay and delay to the ground surface. This effect would be substituted by the high hydraulic conductivity and low specific storage along the casing pipe.

\section{Effect of the insufficient pumping data on the inversion image.}

The inversion scheme proposed by Ozaki et al. (2014) requires the data set of SP variation, the data of quantity of flux and the information of electrical 


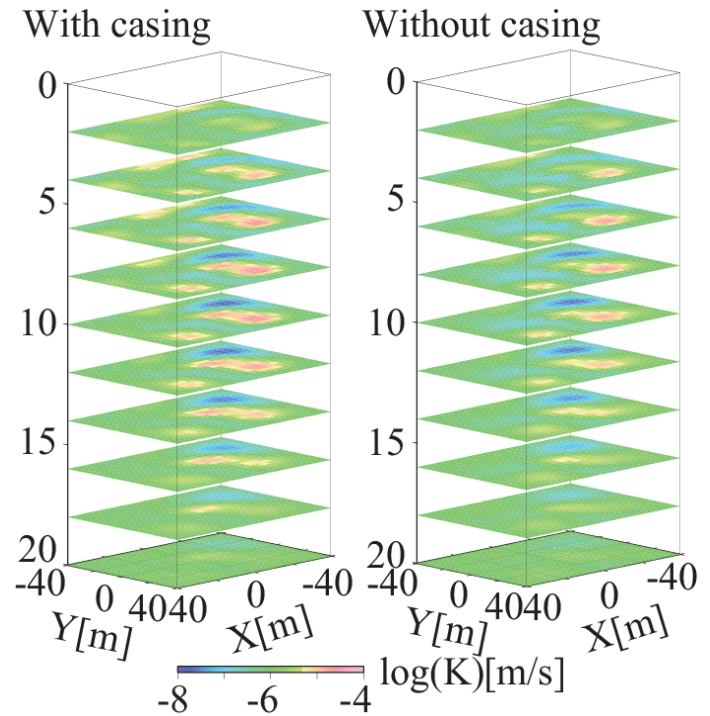

With casing

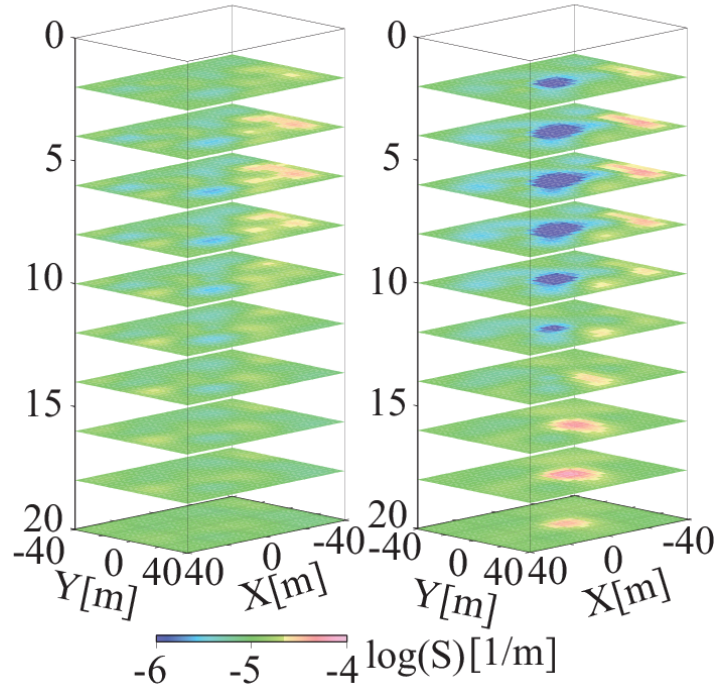

Figure 3 Inversion restults of the SP distribution affected by the metal casing. Hydraulic conductivity (upper) and specific storage (lower) images are shown. In each pictures, the left image is the inversion result considering the effect. The right images shows the results when the casing effect is ignored.

conductivity structure. In these data, the high sampling rate measurement of SP data could be possible because only the measurement of the potential variation on the grand surface is required for the SP observation. However, the observation of the flux data with high sampling rate would be difficult compared to the SP measurement, especially when the monitoring data is focused on. The flux data would be insufficient and the sampling rate of flux data would be lower than that of SP data in such a case.

Considering the difference of difficulty of the measurements discussed in last paragraph, we assume the condition that the sampling rate of the flux data is much lower than that of SP data in this section. When the data is insufficient, the interpolation approach is often used. The spline interpolation is applied to match the sampling rate of flux data with that of SP data in this study. The inversion results with the interpolated flux data are compared with the result with the full flux data.

Figure 4(a) shows the synthetic flux data and one of SP data for the inversion tests. The 36 SP data that the sampling rate is same as that in Figure 4(a) are used for the inversion.

The original flux data is divided into 10 or 5 sections uniformly. The averages in each section are calculated and these averages 10 or 5 averages data are up sampled with spline interpolation (Figure 4(b)). The inversion of SP variations with the interpolated flux data from 10 and 5 data are performed as case 1 and case 2 .

Figure 4(c) shows the input and reproduced SP profiles from the estimated model and input flux data. The calculated SP variation matches with the input SP data when the full flax data is input. Not only the rough trend but also detailed fluctuation of SP variation is reproduced in this case. The detailed fluctuations of reproduced SP variation disappear as the sampling rate of flux data for the interpolation become lower. In such cases, only rough trends appear in the SP variations.

Figure 5 shows the estimation results of the inversion test to check the effect of lack of flux data on the estimation image. All estimation images of deep zone are not clear because the depth of pumping point is shallow and only $6 \mathrm{~m}$. The deep zones are not sensitive in these cases. When the full flux data is used, both the hydraulic conductivity and specific storage structures are reproduced well.

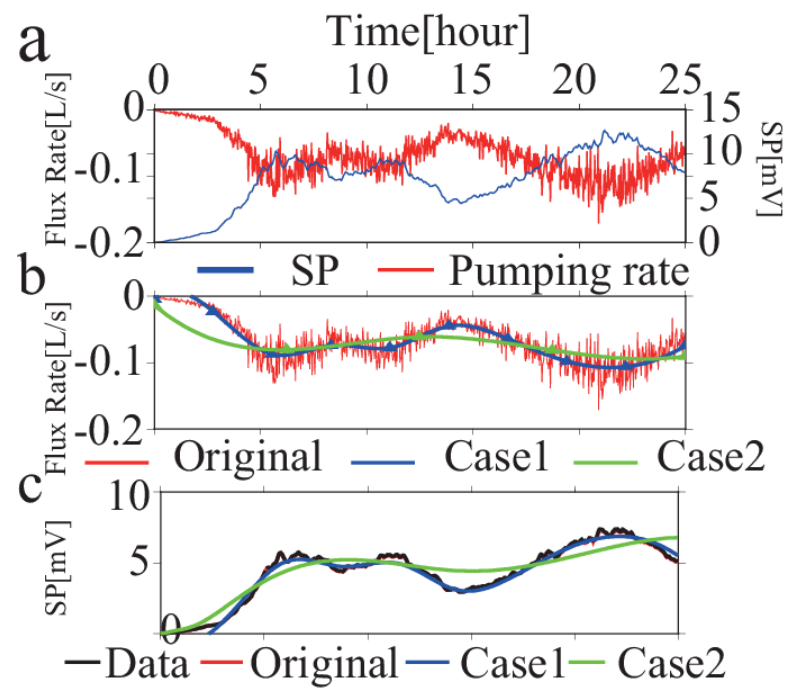

Figure 4 (a) The original snnthetic flux data (red line) and one of SP variation following to the flux (blue line). (b) The flux data for the inversion tests. The original data, interpolated data in case 1 and 2 are shown by red, blue and green lines, respectively. (c) The input SP data and calculated SP variation from the inversion results. The black line shows the input data. One of the reproduced SP variations in case 1 and case 2 are shown by blue and grenn lines, respectively. 


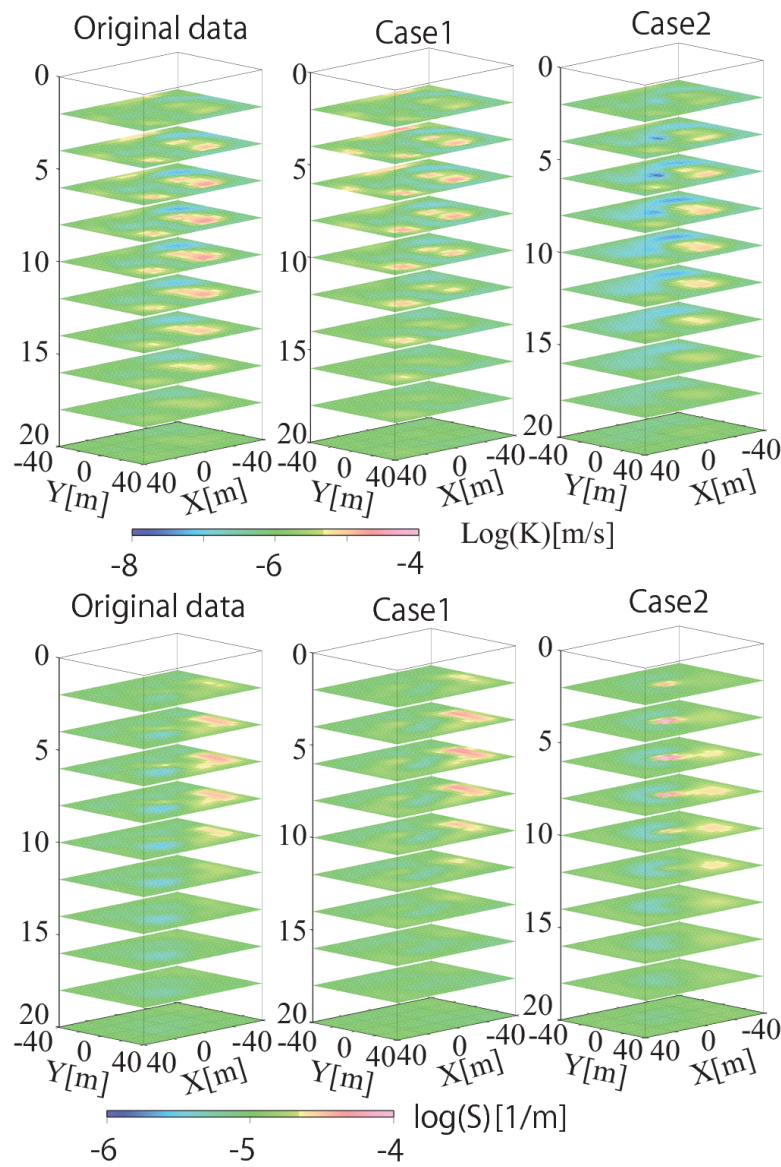

Figure 5 The inversion results of hydraulic conductivity structure. The results when the full flux data is used, case 1 and case 2 are shown from left to right (upper). The inversion results of specific storage structure. From the left, the results with full flux data, in case 1 and in case 2 are shown from left to right (lower). The pumping point is $6 \mathrm{~m}$ depth in this study.

The results of hydraulic conductivity and specific storage structures in case 1 are also very similar to the true models. In this case, the input flux data has the almost same trend as the original flux data. Only the very high frequency components of SP data are not inverted in this case. Such very high frequency components do not affect the global images. As a result, estimated images in case1 would be very similar to the results with full flux data. In the estimation image of hydraulic conductivity structure in case2, the estimation image has very low resolution. However, the estimated image catches the almost same characteristics of true model. On the other hand, the estimated image of specific storage structure does not reproduce the true specific storage structure at all. From this result, only the hydraulic conductivity structure can be reliable when the sampling rate of input flux rate is too low.

\section{CONCLUSION}

In this study, we evaluate the reliability of estimated images of hydraulic conductivity and specific storage structures by the transient SP inversion under two conditions by the numerical tests. Two situations that the casing pipe is existence and the sampling rate of flux data is insufficient are assumed. These results of numerical tests brought us the following conclusions:

- The metal casing pipe changes the estimated hydraulic conductivity and specific storage around the casing pipe to the higher and lower values than true value, respectively if the high conductive effect is ignored.

- The estimated hydraulic conductivity structure is reliable to a certain degree even if the sampling rate of original flux data for the inversion is very low.

The effect of high conductive casing pipe would be possible to be considered by the methods, for example, proposed in this study. The algorithm that estimates both the subsurface structure and flux rate simultaneously would help to improve the problem of low sampling rate of the flux data for the inversion.

ACKNOWLEDGMENT: We are grateful to the Japan Society for the Promotion of Science (JSPS) for the Grants-in-Aid for Science Research in the Research Fellowship program that supported the present study (JSPS: 24.3701).

\section{REFERENCES}

1) Darnet, M., Marquis, G., Sailhac, P., and Gerard, A., 2002, Using Surface SP to Monitor Underground Fluid Flow - an Example from a HDR Stimulation, 64th EAGE Conference and Exhibition, Florence, Italy, Expanded abstract.

2) Deutsch, C., and Journel, A, 1992, GSLIB: Geostatistical Software Library and User's Guide, Oxford Univ. Press, New York.

3) Ozaki, Y., Mikada, H., Goto, T., and Takekawa, J., 2014, The 3D self-potential inversion for the estimation of hydraulic parameters, SEG 2014 Annual Meeting, SEG, Expanded abstracts, 4539-4543, doi: 10.1190/segam2014-1104.1

4) Soueid Ahmed, A., Jardani, A., Revil, A., and Dupont, J. P., 2014, Hydraulic conductivity field characterization from the joint inversion of hydraulic heads and self-potential data, Water Resour. Res., 50, 3502-3522, doi: 10.1002/2013WR014645

5) Titov, K., Revil, A., Konosavsky, P., Straface, S., and Troisi, S., 2005, Numerical modelling of self-potential signals associated with a pumping test experiment, Geophys. J. Int., 162,641-650, doi: 10.1111/j.1365-246X.2005.02676.x 